\title{
Die Friedrich Schlegel-Gesellschaft
}

Die Friedrich Schlegel-Gesellschaft wurde am I 3.4.2007 in Mainz gegründet. Sie setzt sich für die Bewahrung und wissenschaftliche Erforschung der Werke Friedrich Schlegels, den interdisziplinären und internationalen wissenschaftlichen Austausch der erreichten Forschungsergebnisse und für die Förderung des Verständnisses der Werke ein.

Forschungsergebnisse und Tagungsberichte werden im Athenäum - Jahrbuch der Friedrich Schlegel-Gesellschaft und einer eigenen Buchreihe veröffentlicht. $\mathrm{Zu}$ den Arbeitsschwerpunkten der Gesellschaft zählen außerdem die Edition und Kommentierung von Werkausgaben (insbesondere der Kritischen Friedrich-SchlegelAusgabe), die Lehr- und Vortragstätigkeit, wissenschaftliche Veröffentlichungen von Mitgliedern und schließlich die Verbreitung der Kenntnisse über Werk und Person Friedrich Schlegels in der Öffentlichkeit.

Die Friedrich Schlegel-Gesellschaft vertritt die Interessen des wissenschaftlichen Fachpublikums, richtet sich aber auch an interessierte Laien, Schüler, Studenten, Lehrer und Journalisten. Sie bemüht sich um die internationale Vernetzung der Friedrich Schlegel-Forschung.

Weitere Informationen unter der Adresse: www.schlegel-gesellschaft.de 